\title{
One step behind to step ahead - femoral approach to stabilize and to extract functional pacing lead to regain venous access
}

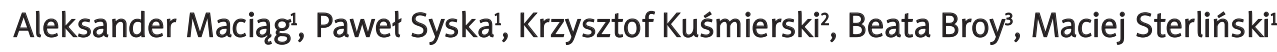 \\ $1^{\text {nd }}$ Department of Coronary Artery Disease, Institute of Cardiology, Warsaw, Poland \\ 2Department of Cardiosurgery and Transplantology, Institute of Cardiology, Warsaw, Poland \\ ${ }^{3}$ Department of Anesthesiology, Institute of Cardiology, Warsaw, Poland
}

Postep Kardiol Inter 2013; 9, 3 (33): 310-312

DOI: 10.5114/pwki.2013.37519

\begin{abstract}
Transvenous lead extraction can be a method to regain venous access. We present the case of a man, aged 67, with indications to upgrade an ICD to a resynchronization therapy device. Since innominate vein occlusion was diagnosed and extraction of an abandoned ventricular pacing lead did not provide lumen regain, a functional atrial lead was extracted with the femoral approach to stabilization and venous access was regained. Asymptomatic vein wall damage but no other complications were recorded. The simultaneous application of different techniques to regain venous access may allow success of the final procedure in system upgrading.
\end{abstract}

Key words: lead extraction, vein occlusion.

\section{Introduction}

The increasing number of patients with cardiac implantable electronic devices (CIEDs) and the prolongation of their survival cause a rise in the absolute percentage of individuals qualified for a transvenous lead extraction (TLE) due to treatment-related complications. There may occur leadrelated occlusion of major venous vessels in up to $50 \%$ of patients. Beside system infection, a rare, but clinically significant class I indication for TLE is regaining the venous access for the implantation of additional and/or new leads. This kind of indication accounts for about $2-5 \%$ of all TLE procedures performed currently in Polish sites [1, 2]. The technique of two opposite venous vessels approach, with removal of the functioning lead, may be used in some individual cases.

We present a case report which illustrates the successful outcome of such a procedure.

\section{Case report}

A 67-year-old man, with postinfarction left ventricular dysfunction and paroxysmal complete atrioventricular block, treated with permanent DDD pacing since 2009 and a dual-chamber implantable cardioverter-defibrillator (ICD DR) since 2011, was referred to upgrade the system for a car- dioverter-defibrillator with resynchronisation therapy (CRT-D) due to heart failure aggravation with gradual ejection fraction decrease to $15 \%$. There were found: the atrial electrode (2009), defibrillation lead (2011) - both active in the ICD system - and an inactive abandoned ventricular pacing lead (2009), secured in the tissues in 2011. All the electrodes were inserted via the left subclavian vein; the device was placed in a subcutaneous pocket in the subclavian area. During the venography complete occlusion of the left anonymous/subclavian vein was discovered. The patient was qualified for abandoned ventricular lead removal to recapture the venous access, needed for the left ventricle (LV) lead implantation. The procedure was performed in a hybrid operation theater in general anesthesia and with temporary endocardial pacing via the femoral vein.

After surgical leads' preparation the locking stylet (Liberator ${ }^{\circledR}$ Beacon ${ }^{\circledR}$ Tip Locking Stylet, Cook Medical Inc.) was inserted into the distal, cardiac part of the abandoned lead and mechanical telescopic sheaths were advanced out of the lead (Byrd Dilator ${ }^{\circledR}$ sheaths, Cook Medical Inc.). However, the relatively weak adhesion between the tissue and electrode, even with moderate traction applied, caused the complete removal of the lead before the sheath reached the other side of the occlusion. This was confirmed by contrast

\section{Corresponding author:}

Maciej Sterliński MD, PhD, 2nd Department of Coronary Artery Disease, Institute of Cardiology, 1 Spartańska St, 02-637 Warsaw, Poland, tel.: +48 2234340 48, e-mail: msterlinski@ikard.pl

Received: 29.04.2013, accepted: 28.06.2013. 


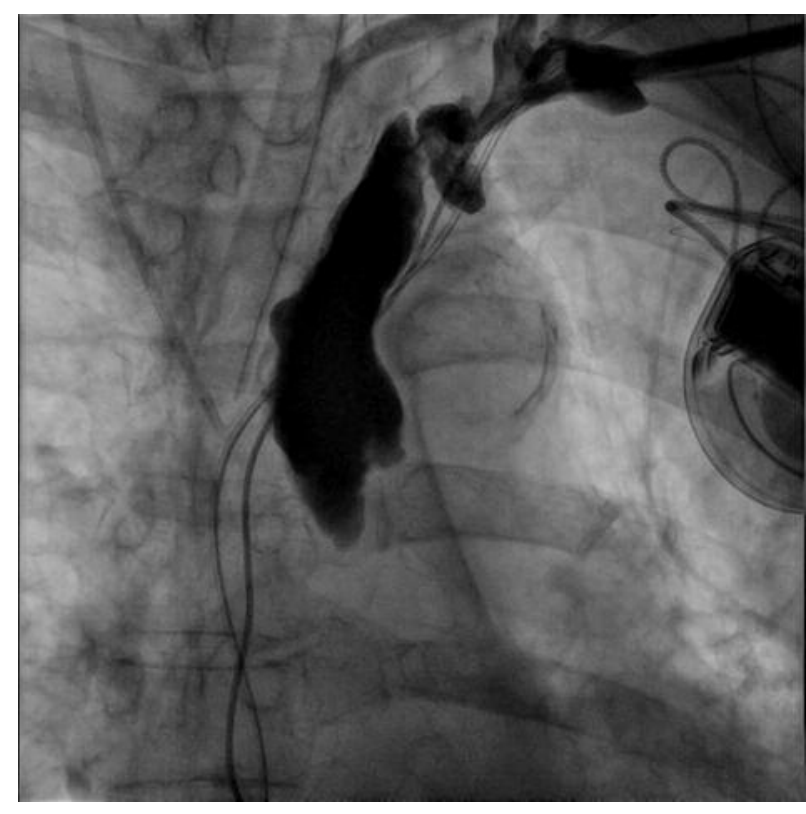

Fig. 1. Fluoroscopy. Anterior-posterior view. Contrasting at the level of the left subclavian vein by Byrd dilator, after abandoned lead removal. Venography reveals anonymous vein wall damage with contrast flow to mediastinum and no connection with vena cava superior

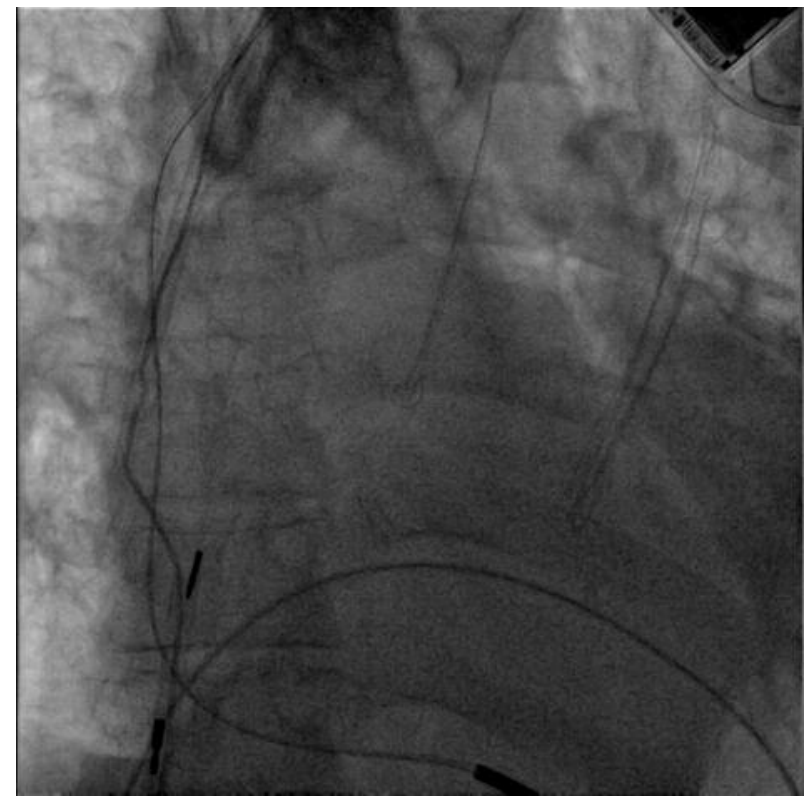

Fig. 3. Fluoroscopy. Anterior-posterior view. Byrd dilator sheath introduced to right atrium by atrial leadrail stabilized by femoral approach. Femoral sheath and Byrd dilator sheath do not contact each other to avoid cutting effect

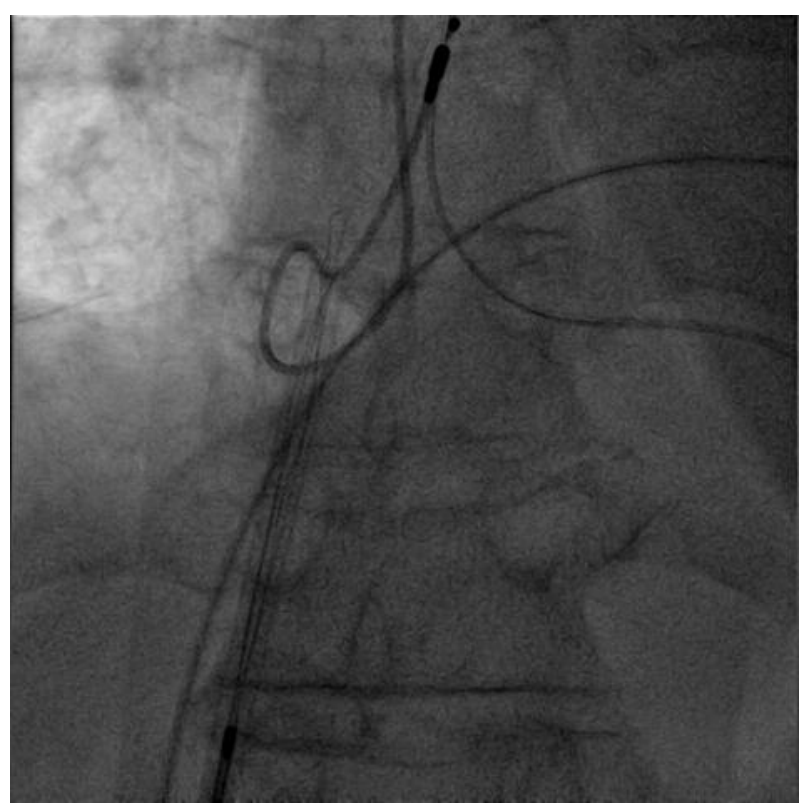

Fig. 2. Fluoroscopy. Anterior-posterior view. Atrial lead caught by Needle's Eye Snare and pulled into the femoral station to produce lead tension

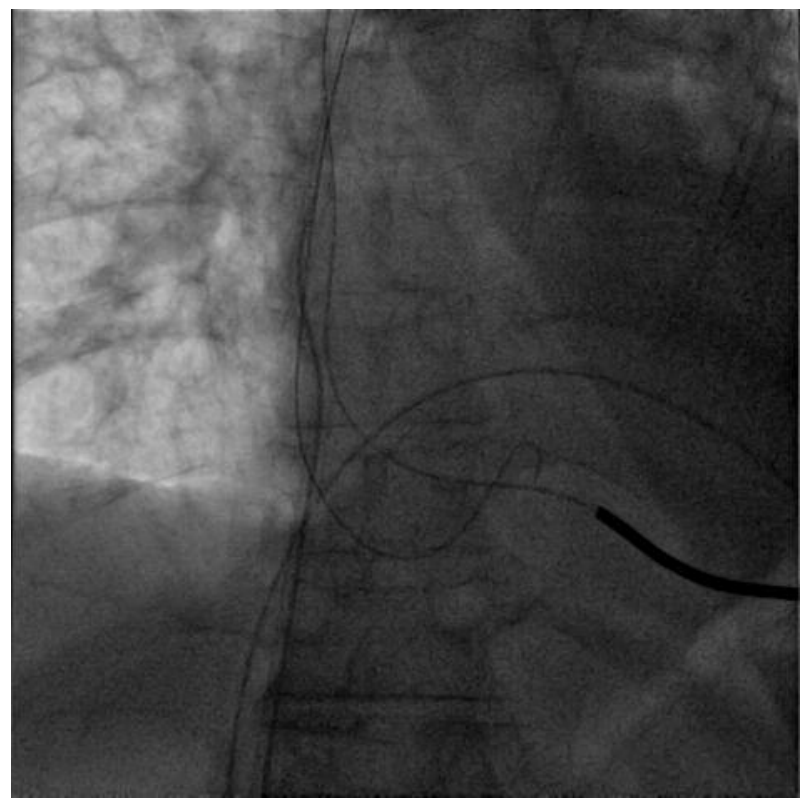

Fig. 4. Fluoroscopy. Anterior-posterior view. The 0.035 " leader introduced into the right atrium/ventricle by Byrd dilator sheath, passing by the atrial lead: subclavian-cardiac approach regained 
injection (Figure 1) and anonymous vein wall damage with contrast flow into the mediastinum occurred as well. The operators decided to continue the procedure due to no symptoms and no accompanying hemodynamic compromise.

Because of the failure of recapture of venous access, it was decided to use and sacrifice the active atrial lead. To avoid further failure, it was decided to fix the atrial lead with countertraction: the sheet and self-locking device station Needle's Eye Snare ${ }^{\circledR}$ (Cook Medical Inc.) was introduced to the inferior vena cava through the right femoral approach, whereby the loop of the lead was captured in the right atrium (RA), released from the atrial appendage and drawn into the guiding catheter (Figure 2). Effective tension to the electrode was applied and telescopic sheaths were advanced. When the level of the RA was reached and mobility of the lead confirmed, the lead was cut in the proximal part and removed through the femoral vein. Through the anonymous vein occlusion, two 0.035 " wires were introduced (Figures 3 and 4). The new active fixation atrial lead and the left ventricular lead to the posterolateral vein were implanted. Acceptable pacing and sensing parameters were confirmed and the procedure was finished typically. After $24 \mathrm{~h}$ stay in the postoperative intensive care unit and 4 days more in the hospital, the patient was discharged home in good functional status. The observation and control tests confirmed the correct function of the CRT-D.

\section{Discussion}

The procedures of CIED therapy performed nowadays more and more often involve system upgrading and thus result in the necessity for implantation of new or additional leads $[1,2]$. An obstacle which may impede the procedure is subclavian vein occlusion. The symptomatic form of this complication occurs in $0.35-3.5 \%$ of patients [3, 4], while asymptomatic blood flow limitation is found in up to $50 \%$ of patients [5]. The successful upgrade of the system may require different techniques [6-8]. One of them is lead extraction with the use of mixed subclavian and femoral access. Both methods are commonly used; the femoral approach is usually performed for unsuccessful subclavian TLE procedures, but some centers prefer it as a method of first choice [2, 9].

The key point of the above-presented procedure was rare, simultaneous use of different lead extraction tools and the main goal of the intervention, which was not only lead removal. The attempted subclavian TLE approach showed inadequate anchoring of the leads. The first electrode was removed "too easily", before the subclavian vein occlusion was overcome with the extraction sheaths. It confirmed indirectly that the risk of loss of the venous access was too big to use a subclavian approach to extract the second lead, which was exactly of the same time from implantation. The femoral access was then introduced to stabilize the remaining lead in the superior vena cava and to allow overcoming the subclavian vein occlusion with the cutting sheaths. It should be emphasized that due to temporal failure of the procedure, extraction of the entirely efficient lead was performed in order to achieve final success of the intervention.

The presented method can be applied in several modifications, using different extraction tools introduced via the femoral approach. The available data confirm the high effectiveness and safety of the technique $[2,6,7,9]$. Some reports show a longer fluoro- and procedure time in femoral access [10], but sometimes it is the only solution.

The current guidelines place the above-described TLE indication in class Ila, level of evidence C [11]. It seems that the number of patients with similar indications for TLE will be increasing in coming years.

The simultaneous application of different intravascular techniques to regain venous access may allow final procedure success in patients who require upgrading of cardiac implantable electronic systems.

\section{References}

1. Chudzik M, Kutarski A, Mitkowski P, et al. Endocardial lead extraction in the Polish Registry - clinical practice versus current heart rhythm society consensus. Arch Med Sci DOI: 10.5114/aoms.2013.33434.

2. Kuśmierski K, Syska P, Maciąg A, et al. Regaining venous access for implantation of a new lead. Postep Kardiol Inter 2013; 9: 16-21.

3. Kar AK, Ghosh S, Majumdar A, et al. Venous obstruction after permanent pacing. Indian Heart J 2000; 52: 431-433.

4. Barakat K, Robinson NM, Spurrell RA. Transvenous pacing leadinduced thrombosis: a series of cases with a review of the literature. Cardiology 2000; 93: 142-148.

5. Sticherling C, Chough SP, Baker RL, et al. Prevalence of central venous occlusion in patients with chronic defibrillator leads. Am Heart J 2001; 141: 813-816.

6. Fisher A, Love B, Hansalia R, et al. Transfemoral snaring and stabilization of pacemaker and defibrillator leads to maintain vascular access during lead extraction. Pacing Clin Electrophysiol 2009; 32: 336-339.

7. Staniforth AD, Schilling RJ. Reuse of occluded veins during permanent pacemaker lead extraction: a new indication for femoral lead extraction. Indian Pacing Electrophysiol J 2002; 2: 97-103.

8. Jarwe $M$, Klug D, Beregi JP, et al. Single center experience with femoral extraction of permanent endocardial pacing leads. Pacing Clin Electrophysiol 1999; 22: 1202-1209.

9. Bracke FA, Dekker L, van Gelder BM. The Needle's Eye Snare as a primary tool for pacing lead extraction. Europace 2013; 15: 1007-1012.

10. Bordachar P, Defaye P, Peyrouse E, et al. Extraction of old pacemaker or cardioverter-defibrillator leads by laser sheath versus femoral approach. Circ Arrhythm Electrophysiol 2010; 3: 319-323.

11. Wilkoff BL, Love CJ, Byrd CL, et al. Transvenous lead extraction: Heart Rhythm Society expert consensus on facilities, training, indications, and patient management: this document was endorsed by the American Heart Association (AHA). Heart Rhythm 2009; 6: 1085-1104. 